YVONNE FRANZ and CHRISTIANE HINTERMANN (eds.)

UNRAVELLING COMPLEXITIES

UNDERSTANDING PUBLIC SPACES 
ISR-FORSCHUNGSBERICHTE

HERAUSGEGEBEN VOM

INSTITUT FÜR STADT- UND REGIONALFORSCHUNG

HEFT 44

REDAKTION: YVONNE FRANZ and CHRISTIANE HINTERMANN

VERLAG DER ÖSTERREICHISCHEN AKADEMIE DER WISSENSCHAFTEN WIEN 2017 
YVONNE FRANZ and CHRISTIANE HINTERMANN (eds.)

\section{UNRAVELLING COMPLEXITIES}

Understanding Public Spaces 
This publication is made possible by a grant from City of Vienna,

MA 7 - Kultur und Wissenschaft, Referat Wissenschafts- und Forschungsförderung

\section{WUIENTR}

Umschlagbild: Urbanität und Lebendigkeit im öffentlichen Raum am Donaukanal, Wien (Foto: Anja Petrovic 2015)

Die Arbeit unterliegt ausschließlich der Verantwortung des ISR und wurde der phil.-hist. Klasse nicht vorgelegt.

Die Abbildungen in dieser Publikation wurden von den jeweiligen AutorInnen zur Verfügung gestellt. Das ISR geht davon aus, dass die AutorInnen die Verwendung und Rechte der Abbildungen geklärt haben.

ISBN 978-3-7001-8133-0

DOI: 10.1553/ISR_FB044

Medieninhaber und Herausgeber: Österreichische Akademie der Wissenschaften

Institut für Stadt- und Regionalforschung, Postgasse 7, A-1010 Wien

Telefon $+43151581 / 3520-3532$

Telefax $+43151581 / 3533$

Redaktion: Yvonne Franz, und Christiane Hintermann

Layout: Florian Partl

Druck: Novographic, A-1230 Wien 